\title{
Spin label saturation transfer ESR studies of protein-lipid interactions in Photosystem II-enriched membranes
}

\author{
Gang Li ${ }^{1}$, Lászió İ. Horváth ${ }^{2 *}$, Peter F. Knowles '. Denis J. Murphy " \\ and Derek Marsh ${ }^{2}$

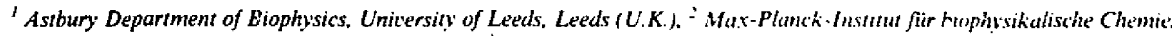 \\ Abteilung Spektroskopie, Güttingen (F.R.G.) and "Department of Biological Scienc'es. Durham ('ntitersity. Dirham (U.K.) \\ Received 12 July 1989 )
}

Key words: Lipid-protein interaction; Photosystem Il; Spin label; Saturatuon transfer ESR: ESR

Saturation transfer ESR has been used to study the Jynamic behaviour of lipids in the appressed regions of thylakoid membranes from pea seedlings. Four difierent phosgno- and galacto-lipid spin latels (phosphatidylcholine tabelled at the 12 or 14 C-atom positions of the $s n-2$ chain, phosphatidylglycerol labelled at the 14-position of the $\mathrm{sm} 2 \mathrm{shaiii}$, and monogalactosyldiacylglycerol labelled at the 12 -mosition of the $s m-2$ chain) were used to probe the lipid environment in phontnsystem H-eiriched membranes prepared by detergent extraction. The ESR spectra show that the majority of the lipid in these preparations is strongly motionally restricted. Values for the effective rotational correlation times of the labelled chains were deduced from the lineheight ratios and integrals of the saturation transfer ESR spectra. The effective rotational correlation times were found to be in the $i^{5} s$ range, indicating a very low lipid chain mobility which correlates with the low lipid content of these preparations. Comparison of the effective rotational correlation times deduced from the different diagnostic regions of thi spectrum revealed little anisotropy in the chain mobility. indicating that the dominant motional mode was trans-gauche isomerization. The effective rotational correlation times deduced from the spectral integrals were similar to those deduced from the lineheight ratios, consistent with the absence of any appreciable fluid lipid component in these preparations. The results also indicate some selectivity of interaction between the lipid species, with phosphatidylcholine exhibiting appreciably slower motion than either phosphatidylglycerol or monogalactosyldiacy lglycerol.

\section{Introduction}

The thylakoid membrane sistem of green plants is the site of all the photosynthetic light reactions (see, for example, Ref. 1). Thylakoids appear to consist of a

\footnotetext{
* Permanent address: Institute of Biophysics. Biological Research Centre, Szeged, Hungary.

Abbreviations: $n$-PCSL, 1-acyl-2-[n-(4.4-dimethyl- $N$-oxyl)stearoyl]sh-glycero-3-phosphocholine; 14-PGSL, 1-acyl-2-[14-(4,4-dimethyl- $N$ oxyl)stearoyl)-sn-glycero-3-phosyhoglycerol: 12-MGDGSL, 1-oleoyl2-[12-(4,4-dimethyl- $N$-oxyl)stearoyl]-sn-glycero-3-galactose: EDTA. ethylenediamine tetraacetic acid; Hepes, $N$-(2-hydroxyethyl)piperazine- $N^{\prime}$-2-ethanesulphonic acid; MES, 2-( $N$-morpholino $)$ thanesulphonic acid; PS II, Photosystem II; PS I, Photosystem I: LHC II. light-harvesting complex I1; STESR, saturation transfer ESR; V, first harmonic ESR absorption signal detected in-phase with respect to the field modulation: $V_{2}^{\prime}$. second harmonic ESR absorption signal detected $90^{\circ}$ out-of-phase with respect to the field modulation.
}

Correspondence: P.F. Knowles, Astbury Department of Biophysics. University of Leeds, Leeds LS2 9JT. U.K. series of stacked membranes which are either appressed' or 'non-appressed'. Although laterally contiguous, the appressed and non-appressed regions differ in their protein [1-3] and possibiy in their lipid [4.5] compositions. The Photosystem II (PS II) protein-pigment zonplex is largely found in the appressed regions, whereas the Photosystem I and ATP synthetase complexes are found mainly in the non-appressed regions. The finding by some authors of lateral heterogeneity in the distribution of acyl lipids in thylakoid membranes may imply that there are specific associations between certain lipids and the heterogeneously distributed protein complexes such as PS I and PS II.

The acyl lipid population of thylakoid membranes is unusual, compared with that of membranes of non-plant origin, in the preponderance of neutral, polyunsaturated galactolipids, which make up $75 \%$ of the total lipids [1.6]. The remainder of the thylakoid lipid is composed largely of more saturated, anionic sulpholipids or phospholipids. Various roles have been proposed for these lipids in the stabilization of thylakoid structure [1,6.7] 
or in the optimization of its photosynthetic function [1]. The possibility of specific lipid-protein interactions has been addressed both in membrane reconstitution studies, and in investigations of the effects of lipolytic cleavage on membrane function. The conflicting conclusions reached by these studies (reviewed in Refs. 1 and 6) point to the need for direct physical measurements on the protein-lipid associations.

The hydrophobic region of the thylakoid lipid bilayer acis as a channel for the passage of the elect ron carrier, plastoquinone, between Photosystem II and the cytochrome $b / j$ complex $[1,8]$. The diffusior: of plastoquinone/plastoquinol through the thylakoid membrane and/or the reoxidation of plastoquinol is believed to be the rate-limiting step in photosynthetic electron transport. The nature and influence of the hydr'phobic core of the thylakoid membrane therafne cieserves further attention. It is generally agreed that the fluidity of the lipid chains of thylakoid mem?.)ranes is lower in the appressed than in the non-appressed regions [9-14]. Indeed, it has been found that the lipid chain motion in the hydrophobic regions of the appressed membranes approaches the rigid limit of conventional spin label ESR spectroscopy $[11,15]$

For the above reasons, we have undertaken a series of studies of the lipid chain mobility and lipid-protein interactions in thylakoid membranes and subthylakoid membrane fractions, using as probes spin-labelled galacto- and phospholipids which are structurally similar to their counterparts in thylakoid membranes [16,17]. These studies have shown that much of the lipid in PS II-enriched subthylakoid fractions prepared by detergent extraction is motionally restricted. Since these preparations retain oxygen-evolving capacity, the motional requirements for function merit further investigation. In the present work we have attempted to define the degree of chain motional restriction in greater detail, for the various lipid classes, by using saturation transfer ESR (STESR) spectroscopy. STESR extends the motional limit of conventional spin-label ESR spectroscopy to rotational correlation times in the $10^{-7}$ to $10^{-3} \mathrm{~s}$ range [18] and is therefore well suited to a study of the lipid chain motion in the appressed regions of the thylakoid membrane.

\section{Materials and Methods}

Spin-labelled phospluatidylcholines, $n$-PCSL, were prepared from the corresponding spin-labelled stearic acids and egg lysophosphatidylcholine as described in Ref. 19. Spin-labelled phosphatidylglycerol, 14-PGSL, was prepared from the corresponding spin-labelled phosphatidylcholine by headgroup exchange catalyzed by phospholipase D [19]. Spin-labelled monogalactosyldiacylglycerol, 12-MGDGSL, was a gift from Dr. 1 .
Nishida, National Institute for Basic Biology, Okazaki, Japan, and was prepared as described in Ref. 20.

Pea seeds (Pisium sativum L, var. Kelvedon Wonder) were germinated directly in vermiculite, kept in the dark for the first 5 days at $25^{\circ} \mathrm{C}$, and then transferred to a controlled growth room $\left(20^{\circ} \mathrm{C}, 12 \mathrm{~h}\right.$, light/dark). Leaves were harvested after approximately 14 . days of growth, following a period of $12 \mathrm{~h}$ dark (to reduce starch levels) and $1 \mathrm{~h}$ light. The leaves were homogenized in grinding medium $(330 \mathrm{mM}$ glucose, 50 $\mathrm{mM} \mathrm{Na} \mathrm{HPO}_{4}, 50 \mathrm{mM} \mathrm{K} \mathrm{HPO}_{4}, 5 \mathrm{mM} \mathrm{MgCl}, 25$ $\mathrm{mM} \mathrm{NaCl}$ (p 6.5)) with two bursts of $2 \mathrm{~s}$ in a chilled blender. The homogenate was filtered through eight layers of muslin, and the filtrate centrifuged briefiy (30 s, $2500 \times g$ ) to sediment the chloroplasts. The pellets were then resuspended and vortex mixed gently for $20-60 \mathrm{~s}$ in a small volume of $5 \mathrm{mM} \mathrm{MgCl}$. The stacked thylakoids were then twice washed free of lysed chloroplast envelopes, by further centrifugation after resuspension in $330 \mathrm{mM}$ sorbitol, $2 \mathrm{mM}$ EDTA, $5 \mathrm{mM}$ $\mathrm{MgCl}_{2}, 50 \mathrm{mM}$ Hepes ( $\mathrm{pH}$ 7.6). The final pellets were resuspended in the same buffer and kept in the dark at $4^{\circ} \mathrm{C}$ until further use.

The chlorophyll $a / b$ ratio of both leaves and thylakoid preparations was measured by the method of Arnon [21]. Values for the thylakoid preparations were similar to those for the intact leaves (approx. 2.4). Any preparations with appreciably lower values were discarded. The protein content of the thylakoid preparation was measured by the method of Markwell et al. [22] and the lipid content was determined by gas chromatographic and gravimetric analysis as described in Ref. 23. The lipid/chlorophyll/protein ratio was determined to be $33: 8: 58(w / w)$. The intactness and purity of the thylakoid preparations were also checked by thin section electron microscopy, according to the methods of Telfer et al. [24]. The electron micrographs showed that the preparations were essentially free of contamination by other membranes and organelles.

Photosystem II-enriched membranes were prepared by detergent extraction according to Ref. 25 . Thylakoid membranes were suspended in stacking medium (330 $\mathrm{mM}$ sorbitol, $5 \mathrm{mM} \mathrm{MgCl}_{2}, 15 \mathrm{mM} \mathrm{NaCl}, 50 \mathrm{mM}$ Hepes (pH 7.0)). The suspension was then diluted to a concentration of $2 \mathrm{mg}$ chlorophyll/ml and incubated in the dark for 25 min after addition of Triton X-100 at a concentration of $50 \mathrm{mg} / \mathrm{ml}$. The PS II-enriched membranes were then harvested by centrifugation at 40000 $\times g$ for $30 \mathrm{~min}$ and the membrane pellets resuspended in $300 \mathrm{mM}$ sucrose, $5 \mathrm{mM} \mathrm{MgCl}, 15 \mathrm{mM} \mathrm{NaCl}, 20$ $\mathrm{mM}$ Hepes ( $\mathrm{pH}$ 7.0). The lipid/chlorophyll/protein ratio of the PS Il-enriched membranes was determined to be $16: 17: 67(\mathrm{w} / \mathrm{w})$.

The purity of the PS II membrane preparations was checked by polyacrylamide gel electrophoresis. The gel patterns were enriched in the PS II and LHC II centres, 
but contained very little of the $18-25$ and $70 \mathrm{kDa}$ polypeptides characteristic of the PS I reaction centres. Thus there was little cross-contamination of the two photosystem complexes. The lipid compositions of the thylakoid and of the PS II membranes were determuned as described previously [4].

For spin-labelling, PS II-enriched membranes comprising approx. $2 \mathrm{mg}$ of polar lipids were suspended in 2-3 ml of $20 \mathrm{mM} \mathrm{Mes,} 50 \mathrm{mM} \mathrm{KCl}, 5 \mathrm{mM} \mathrm{MgCl} 2$ (pH 6.5 ) and $20 \mu \mathrm{l}$ of $1 \mathrm{mg} / \mathrm{ml}$ spin label solution in ethanol was added slowly. The sample was then vortex mixed briefly and ircubated for $15-20 \mathrm{~min}$ in the dark at room temperature. The spin-labelled sample was centrifuged $\left(45 \mathrm{~min}, 90000 \times \mathrm{g}, 4^{\circ} \mathrm{C}\right.$ ) and washed in 13 $\mathrm{ml}$ of the same buffer, using a Beckmann SW-40 swingout rotor. The pellet from the final centrifugation was transferred with about $10 \mu \mathrm{l}$ of buffer to a $100 \mu \mathrm{l}$ capillary $(1 \mathrm{~mm}$ i.d.) sealed at one end. The sample was then packed using a bench-top centrifuge, excess buffer removed, the sample trimmed to $5 \mathrm{~mm}$ in length, and the capillary flushed with argon prior to sealing. Argon-flushed buffers were used throughout the sample preparation and the capillary tubes were wrapped with aluminium foil to mirimue light-induced ESR signals.

ESR spectra were recorded on a Varian Century Line $9 \mathrm{GHz}$ spectrometer interfaced to a PDP $11 / 10$ computer for data collection. The $5 \mathrm{~mm}$ samples in the 1 $\mathrm{mm}$ I.D. capillaries were carefully centered in the TE102 rectangular cavity, following the protocol of Ref. 26 . The capillaries were accommodated within a standard 4 $\mathrm{mm}$ quartz ESR tube which contained light silicone oil for thermal stability. Temperature was regulated using a nitrogen gas flow system. Conventional in-phase absorption spectra ( $V_{t}$ display) were recorded at a modulation amplitude of $1.6 \mathrm{G} \mathrm{p}$-p, at the same microwave power as used for the STESR experiments. STESR spectra were recorded in the second harmonic, $90^{\circ}$ out-of-phase, absorption mode ( $V_{2}^{\prime}$ display) at a modulation frequency of $50 \mathrm{kHz}$ and with a modulation amplitude of $5 \mathrm{G}$ p-p. All STESR measurements were performed at a microwave power which gave an average $H_{1}$ field over the sample of $0.25 \mathrm{G}$, according to a standard protocol $[26,27]$. Details of the calibration of the spectrometer, along with rotational correlation time calibrations, are described elsewhere [26.28]. The first integrals of the $V_{2}^{\prime}$ STESR spectra. $I_{\mathrm{ST}}$, were nor-
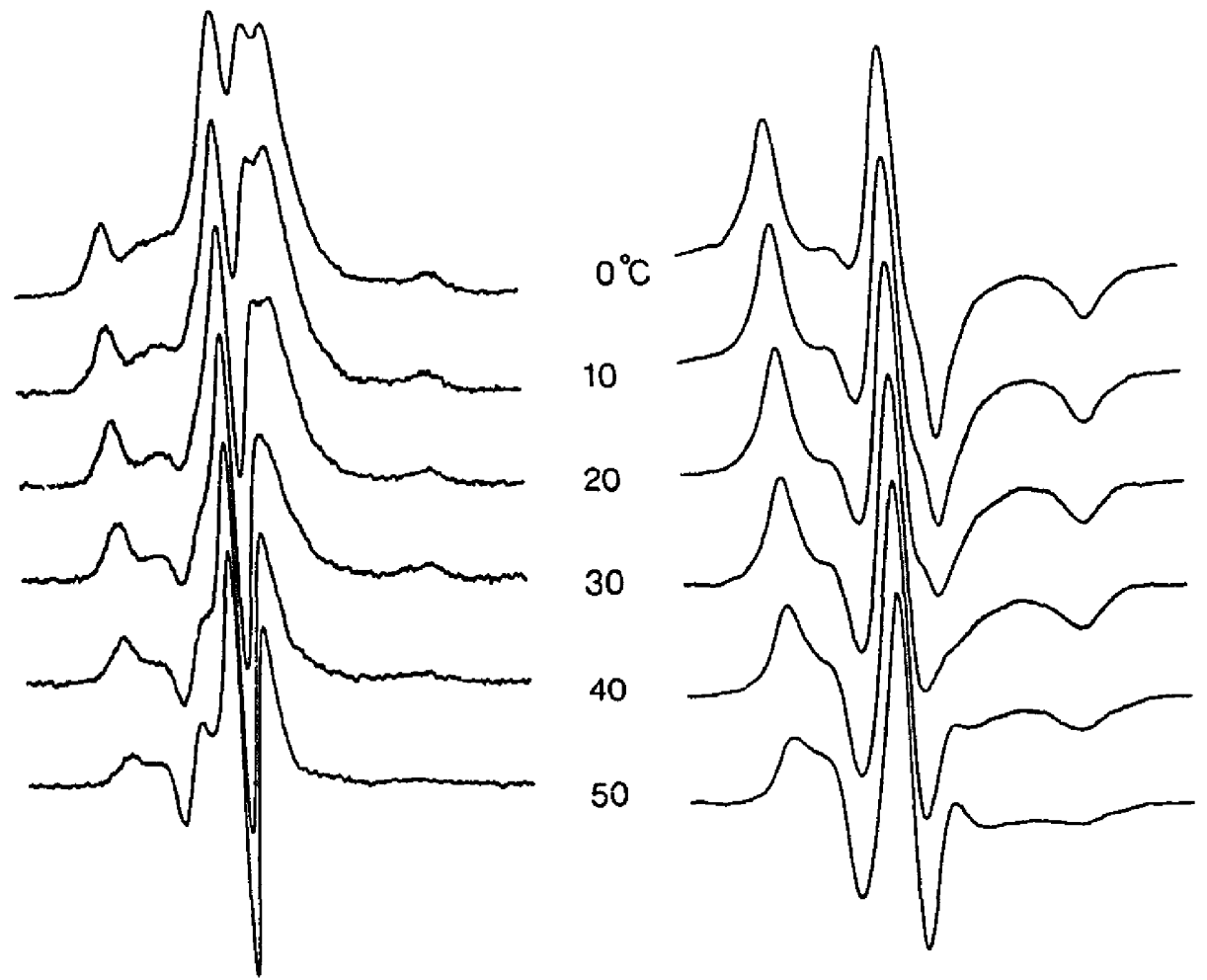

Fig. 1. Temperature dependence of the ESR specta of the 12-MGDGSL monogalactosyldiacylglycerol spin label in PS II-enriched membranes from pea thylakoids. Left-hand side: second harmonic, $90^{\circ}$ out-of-phase, absorption STESR spectra ( $V_{2}^{\prime}$ display). Right-hand side: first harmonic. in-phase, absorption ESR spectra ( $V_{1}$ display). Total scan width $=100$ gauss. 

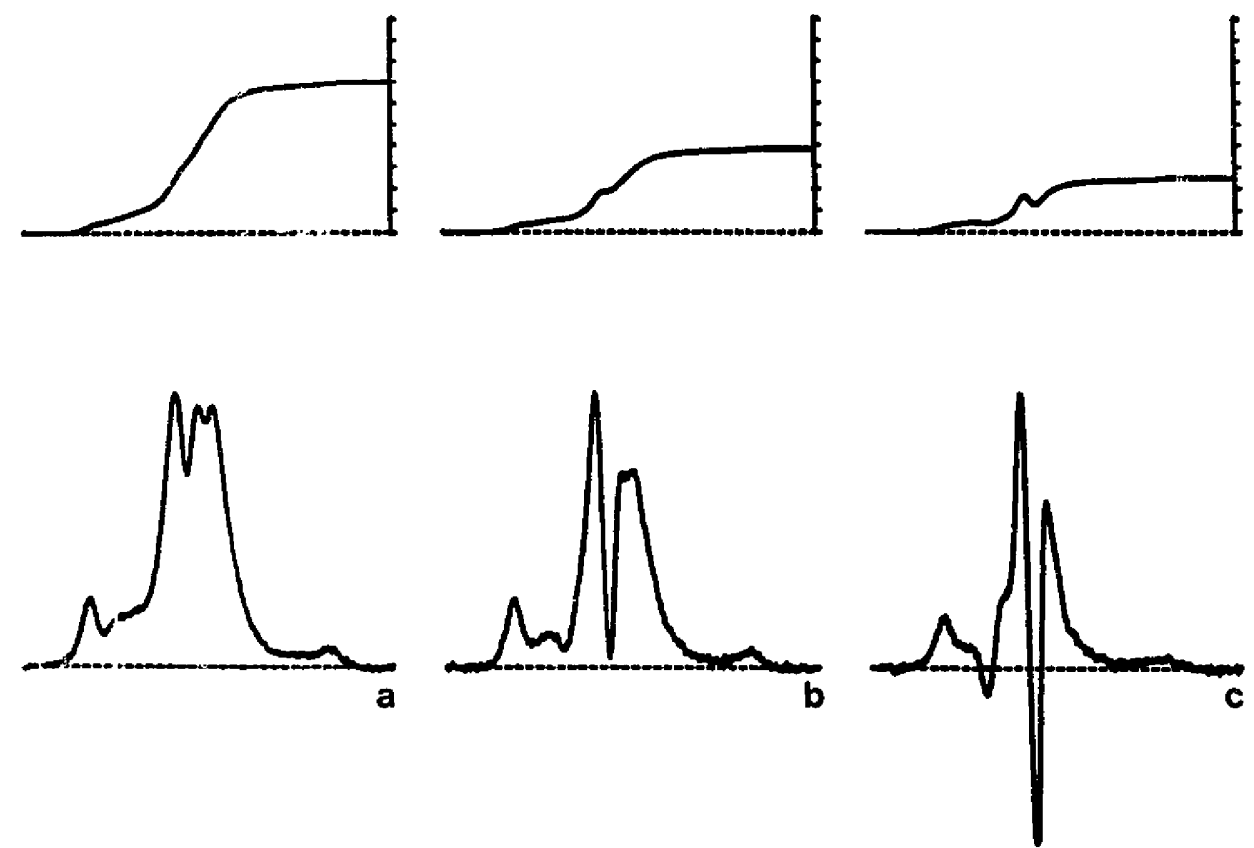

Fig. 2. Lower row: second harmonic. $90^{\circ}$ out-of-phase, absorption ESR spectra ( $\mathrm{V}_{2}^{\prime}$ display) of the 12-MGDGSL monogalactosyldiacylglycerol spin label in PS II-enriched mem' -anes from pea thylakoids. (a) Recorded at $0^{\circ} \mathrm{C}$, (b) at $20^{\circ} \mathrm{C}$, and (c) at $40^{\circ} \mathrm{C}$. Upper row: first integrals of the STESR spectra in the lower row, normalized with respect to the double-integrated intensity of the conventional spectra. (Full scale $=10^{-2}$, correspunding to an effective correlation time of $370 \mu \mathrm{s}$ ). Total scan width $=100$ gauss.

malized with respect to the double integral of the conventional $V_{1}$ spectrum recorded under standard conditions as described in Ref. 29.

\section{Results}

Representative second harmonic $90^{\circ}$ out-of-phase absorption STESR spectra, together with the corresponding conventional ESR spectra, for the 12MGDGSL spin label in PS II-enriched membranes at different temperatures are shown in Fig. 1. Large changes are seen in the STFSR spectra with increasing temperature, whilst the conventional spectra change rel-

\section{TABLE I}

Effective rotational correlaion times, $\tau_{R}^{e f f}$ ( $\mu$ s) deduced from the lineheight ratios $L^{\prime \prime} / L C^{\prime} / C$ and $H^{\prime \prime} / H$ in the low-field, central and high-field regions of the STESR spectrum, respectively, and from the normalized STESR integral, $I_{S T}$, for the I2-MGDGSL spin label in PS II-enriched membranes

\begin{tabular}{|c|c|c|c|c|c|c|}
\hline$\overline{\text { STESR parameter }}$ & $\tau_{R}^{\text {eff }}$ & & & & & \\
\hline Temperature $\left({ }^{\circ} \mathrm{C}\right):$ & & 10 & 20 & 30 & 40 & 50 \\
\hline$\overline{I_{\mathrm{ST}}}$ & 68 & 28 & 15 & 6 & 3.2 & 3.5 \\
\hline$L^{\prime \prime} / L$ & 23 & 15 & 7 & - & - & - \\
\hline$C^{\prime} / C$ & 46 & 13 & 5 & - & - & - \\
\hline$H^{\prime \prime} / H$ & 55 & 5.5 & 14 & - & - & - \\
\hline
\end{tabular}

atively little, except at the highest temperatures measured. The changes observed in the conventional spectra at the higher temperatures are due to a small fluid population of lipids, which comprises approx. $30 \%$ of the total lipids in the PS II-enriched membrane preparations [17]. This fluid component affects the lineshapes of the STESR spectra, making the normal lineheight ratio method of determining the effective rotational correlation times * [18] inappropriate at the higher temperatures. However, the STESR integral method of determining the effective rotationai correlation times, which is applicable to multi-component STESR spectra [29], can be applied at all temperatures.

Evaluation of the STESR integrals is illustrated in Fig. 2. Characteristic changes are seen in the total integral that correspond to decreasing rotational correlation times with increasing temperature. The fast motional fluid lipid spectral component contributes negli-

\footnotetext{
* In the case of anisotropic motion, the correlation times derived from the isotropic haemoglobin calibrations can only be referred to strictly as 'effective' values $[30-34,39]$. The effects of motional anisotropy are manifested in different 'effective' correlation times being obtained from the different diagnostic regions of the STESR spectrum $[30,32,33]$. This point is atdressed further under Discussion.
} 
TABLE II

Effective rotational correlation times, $\tau_{A}^{d f}(\mu s)$, deduced from the normalized STESR integral, $I_{S T}$, for the I4-PGSL, 14-PCSL and 12.PCSL spin labels in PS II-enriched membranes

\begin{tabular}{lrrrrrr}
\hline \begin{tabular}{l} 
Spin label \\
\multicolumn{1}{c}{ Temperature $\left({ }^{\circ} \mathrm{C}\right)$}
\end{tabular} & \multicolumn{2}{l}{$\tau_{\mathrm{R}}^{\text {eff }}(\mu \mathrm{s})$} & & & & \\
\hline 14-PGSL & 10 & 20 & 30 & 40 & 50 \\
\hline 14-PCSL & 59 & 22 & 15 & 10 & 11 & 8 \\
12-PCSL & 97 & 55 & 56 & 11 & 7 & 6 \\
\hline
\end{tabular}

gibly to the total STESR integral and therefore does not interfere with the evaluation of the effective correlation time [29]. The effective rotational correlation times of the spin-labelled lipid chain have been evaluated by comparison with calibration spectra from isotropically rotating spin-labelled haemoglobin [28]. The values deduced from both the normalized STESR integral and the diagnostic lineheight ratios [16] are given for the 12 MGDGSL spin label in Table I. At low temperatures, all four STESR parameters yield effective rotational correlation times of a similar order of magnitude. Some differences are observed between the various parameters that can be attributed in part to the underlying incipient fluid component, and also to the different inherent sensitivity and precision of the parameters. As the temperature is increased these discrepancies get larger and the lineheight ratios are no longer reliable parameters, as explained previously.

The effective rotational correlation times deduced from the normalized STESR integral are given for the three phospholipid spin labels in PS Il-enriched membrane preparations in Table II. As for the 12-MGDGSL label, all phospholipid labels yield values in the $10^{-5} \mathrm{~s}$ regime, indicating that the chain motion is remarkably slow. The values obtained for the two different positional isomers of the phosphatidylcholine spin label are of comparable magnitude, and somewhat larger than for either the 14-PGSL or 12-MGDGSL (Table I) spin labels.

\section{Discussion}

The results from all four spin labels indicate that the chain motion is very slow for the lipids directly associated with the Photosystem I1-protein complex. The effective rotational correlation times ate beyond the limits of motional sensitivity of conventional spin label ESR, indicating that analysis by saturation transfer ESR is essential. Whereas the bulk of the lipids (approx. 60\%) in whole thylakoid membranes is ir. a fluid state $[10,17]$, detergent extraction of the PS II centres yields preparations which are relatively low in lipid content, consisting primarily of the first lipid shell surrounding the protein complex. This preparation therefore provides a system wcli-suited for studying the mobility of the lipids directly aisociated with the protein.

Previous ryeasurements of the exchange rates of lipids at the interface with integral proteins in membranes containing an excess of lipids have yielded off-rates in the region of $10^{7} \mathrm{~s}^{-1}[30,31]$, which is considerably more rapid than the rates of chain rotation observed in the present study. However, in the present case, the population of fluid lipids with which exchange may take place is very limited and therefore exchange is unlikely to make an appreciable contribution to the effective rotational rates measured. It seems likely that excrange may be the predominant contribution to the mobility of first-shell lipids at the protein interface in lipid-rich membranes.

One striking feature of the STESR spectra recorded at the lower temperatures in Figs. 1 and 2 is the high intensity in the centre of the spectrum. This suggests that the chains are not undergoing a preferentially more rapid rotation about their long axes (cf. Refs. 32, 33). This conclusion is borne out by the effective currelation time deduced from the $c^{\prime} / C$ lineheight ratio given in Table I. The reasonable consistency of the zorrelation times deduced from the three different lineheight ratios suggests that the motion must approximate the isotropic rotational diffusion for which the calibrations were deduced. Considering the possible motional modes of the lipid chains, it therefore seems likely that these are dominated by trans-gauche isomerization, rather than a preferential axial rotation, at the lipid/protein interface.

The order of magnitude of the effective correlation times is much greater than that found for the chain rotational motions in the fluid phase of lipid membranes, which are typically in the $10^{-9}$ to $10^{-8} \mathrm{~s}$ range [34,35], and approaches much closer to that obtained in gel phase lipid bilayers [32]. The motions are, however, significantly faster than those of the closely packed chains in lipid gel phases which can have effective correlation times of the order of $10^{-4} \mathrm{~s}$. It is of interest to compare the effective correlation :imes for lipid chain motion with those expected for protein rotational diffusion. Using the hydrodynamic approach of Saffman [36], the correlation time for protein rotation is related to the effective membrane viscosity, $\eta$, by:

$\tau_{R \| \mid}=2 \pi \eta a^{2} h /(3 k T)$

where $h$ is the height of the membrane-spanning region. For a PS II-LHC II protein complex of approximate mean diameter $2 a=133 \AA$ [1], the predicted rotational correlation time is $\tau_{\mathrm{R} \|} \approx 55 \mu \mathrm{s}$, assuming $\eta \approx 5 \mathrm{P}$ [37]. This estimate is compa: $d$ ble to the values given in Table I, however the STESR ، iata do not suggest the characteristic uniaxial anisotropy expected for integral protein rotational diffusion. In addition, it is possible that the 


\section{TABLE III}

Polar lipid composition (molt) of thylakoid membranes and subthylakoid membrane fractions from pea seedlings

MGDG. monogalactosyldiacylglycerol; DGDG, digalactosyldiacylglycerol; PG. phosphatidylglycerol; SQDG. sulphoquinovosyldiacylglycerol; PC. phosphatidylcholine.

\begin{tabular}{llll}
\hline Lipid & Thylakoid & PS I fraction & PS II fraction \\
\hline MGDG & 40.3 & 38.4 & 46.0 \\
TGDG & 25.1 & 28.3 & 22.7 \\
PG & 11.7 & 11.6 & 10.1 \\
SQDG & 13.0 & 12.6 & 11.3 \\
PC & 5.6 & 4.0 & 4.3 \\
Remaining & 4.3 & 5.1 & 5.6 \\
\hline
\end{tabular}

effective viscosity may be considerably higher in complexes with low lipid/protein ratio, and by the same token the lipid motions at the protein interface may be correspondingly slowed down. The detergent-extracted PS II-enriched membranes gave (2,5-dimethyl-p-benzoquinone)-dependent oxygen evolution of 180-190 $\mu \mathrm{mol}$ $\cdot \mathbf{s} \cdot \mathbf{h}^{-1} \cdot(\mathrm{mg} \text { chlorophyll })^{-1}$. This rate was not altered significantly following incorporation of the spin-labelled probes.

Although PS II-enriched membranes have a lower lipid/protein ratio, the distribution of lipid classes is not notably changed from that found in thylakoids (see Table III). Therefore, it is unlikely that there is inhibition as a result of preferential depletion of a single lipid class with a possitle functional role (cf. Ref. 39). In addition, it is found in the present work that all lipid classes display a strong degree of motional restriction of the acyl chains. The question thus arises to what extent this inhibition of the chain motions influences the functional properties of the photosystem II reaction centre. This question is important, since models for thylakoid membrane function (see Ref. 39 for a review) require mobility of protein components, e.g. LHC II, and redox carriers, e.g. plastoquinone. This mobility would be strictly limited at the lipid/protein interface if the lipid acyl chain motion is restricted in this region. Under these circumstances, it is indeed possible that the electron transfer rate may be determined or influenced by the exchange rate at the lipid/protein interface.

\section{Acknowledgements}

We wish to thank Dr. I. Nishida for his kind gift of the 12-MGDGSL spin albel. T.L. acknowledges financial support from the British Council, the Education Ministry of China and the Max-Planck Society.

\section{References}

1 Murphy, D.J. (1986) Biochim. Biophys. Acta 864, 33-94.

2 Andersson, B. and Anderson, J.M. (1980) Biochim. Biophys. Acta $5913,462-472$.
3 Anderson, J.M. (1982) FEBS Lett. 138, 62-68.

4 Murphy, D.J. and Woodrow. I.E. (1983) Biochim. Biophys. Acta 725, 104-112.

5 Gounaris. K.. Sundby. C.. Andersson. B. and Barber, J. (1983) FEBS Lett. 156, 170-174.

6 Murphy, D.J. (1986) in Encyclopaedia of Plant Physiology, New Series (Staehelin, L.A. and Amtzen, C.J., eds.), Vol. 19, pp. 425-428. Springer Verlag. Berlin.

7 Murphy, D.J. (1982) FEBS Lett. 150, 19-26.

8 Millner, P.A. and Barber. J. (1984) FEBS Lett. 169-1-6.

9 Strzalka. K. and Subczynski. W.K. (1981) Photobiochem. Photobiophys. 2, 227-232.

10 Ford, R.C., Chapman, D.J., Barber, J., Peterson, J.Z. and Cox, R.P. (1982) Biochim. Biophys. Acta 681, 145-151.

11 Amonson, H., Waggoner. C., More, J. and Berg, S.P. (1983) Biochim. Biophys. Acta 725, 519-528.

12 Ford, R.C. and Barber, J. (1983) Biochim. Bjophys. Acta 722, 341-348.

13 Millner, P.A., Chapman, D.J. and Barber, J. (1984) Binchim. Biophys. Acta 765, 282-288.

14 Millner, P.A. Chapman, D.J., Mitchell. R.A. and Barber, J. (1984) in Structure, Function and Metabolism of Plant Lipids (Siegenthaler, P.A. and Eichenberger, W., eds.). pp. 433-436. Elsevier, Amsterdam.

15 Waggoner, C., Arnonson, H., More, J. and Berg. S.P. (1985) Photosynth. Res. 6, 57-71.

16 Murphy, D.J. and Knowles, P.F. (1984) in Structure, Function and Metabolism of Plant Lipids (Siegenthaler, P.A. and Eichenberger, W., eds.), pp. 425-428, Elsevier, Amsterdam.

17 Li, G., Knowles, P.F., Murphy, D.J., Nishida, 1. and Marsh, D. (1989) Biochemistry 28, 7446-7452.

18 Thomas, D.D., Dalton, L.R. and Hyde, J.S. (1976) J. Chem. Phys. 65, 3306-3324.

19 Marsh, D. and Watts, A. (1982) in Lipid-Protein Interactions (Jost, P.C. and Griffith, O.H., eds.), Vol. 2, PP, 53-126, Wiley-Interscience, New York.

20 Nishida, I. and Yamada, M. (1985) Biochim. Biophys. Acta 813, 298-306.

21 Arno:1, D.I. (1949) Plant Physiol. 67, 1-15.

22 Markwell, M.A.K., Haes, S.M., Tolbert, N.E. and Bieber, L.L. (1981) Methods Enzymol. 72, 296-303.

23 Murphy, D.J., Cummins, 1. and Kang, A.S. (1989) Biochem. J. 258. 285-293.

24 Telier, A., Nicholson, J. and Barber, J. (1976) FEBS Lett. 65. 77-83.

25 Berthold, D.A., Babcock, G.T. and Yocum, C.F. (1981) FEBS Lett, 134, 231-234.

26 Fajer, P. and Marsh, D. (1982) J. Magn. Reson. 49, 212-214.

27 Hemminga, M.A., De Jager, P.A., Marsh, D. and Fajer, P. (1984) J. Magn. Reson. 59, 160-163.

28 Horváth, L.I. and Marsh, D. (1988) J. Magn. Reson. 80, 314-317.

29 Horváth, L.J. and Marsh, D. (1983) J. Magn. Reson. 54, 363-373.

30 Marsh, D. and Horváth, L.I. (1989) in Advanced EPR in Biology and Biochemistry (Hoff. A.J., ed.), Elsevier, Amsterdam.

31 Marsh, D. (1989) Stud. Biophys., in press.

32 Marsh, D. (1980) Biochemistry 19, 1632-1637.

33 Fajer, P. and Marsh, D. (1983) J. Magn. Reson. 51, 446-459.

34 Lange, A., Marsh, D., Wassmer, K.-H., Meier, P. and Kothe, G. (1985) Biochemistry 24, 4383-4392.

35 Moser, M., Marsh, D., Meier, P., Wassmer, K.-H. and Kothe, G. (1989) Biophys. J. 55, 111-123.

36 Safímann, P.G. (1976) J. Fluid Mech. 73, 593-602.

37 Cherry, R.J. and Godfrey, R.E. (1981) Biophys. J. 36, 257-276.

38 Gounaris, K., Barber. J. and Harwood. J.L. (1986) Biochem. J. 237, 313-326.

39 Polnaszek, D.F., Marsh, D. and Smith, J.C.P. (1981) J. Magn. Res. 43, 54-64. 